\title{
Implementation of image-guided brachytherapy (IGBT) for patients with uterine cervix cancer: a tumor volume kinetics approach
}

\author{
Heloisa de Andrade Carvalho, MD, PhD', Lucas Castro Mendez, MD', Silvia Radwanski Stuart, MD', \\ Roger Guilherme Rodrigues Guimarães, MDl, Clarissa Cerchi Angotti Ramos, MD!, Lucas Assad de Paula, MD², \\ Camila Pessoa de Sales, PhD', André Tsin Chih Chen, MDl, Roberto Blasbalg, MD, PhD², Ronaldo Hueb Baroni, MD, PhD² \\ IRadiotherapy Division, '2Magnetic Resonance Division, Departamento de Radiologia e Oncologia Faculdade de Medicina da Universidade \\ de São Paulo, Brazil
}

\begin{abstract}
Purpose: To evaluate tumor shrinking kinetics in order to implement image-guided brachytherapy (IGBT) for the treatment of patients with cervix cancer.

Material and methods: This study has prospectively evaluated tumor shrinking kinetics of thirteen patients with uterine cervix cancer treated with combined chemoradiation. Four high dose rate brachytherapy fractions were delivered during the course of pelvic external beam radiation therapy (EBRT). Magnetic resonance imaging (MRI) exams were acquired at diagnosis (D), first (B1), and third (B3) brachytherapy fractions. Target volumes (GTV and HR-CTV) were calculated by both the ellipsoid formula (VE) and MRI contouring (VC), which were defined by a consensus between at least two radiation oncologists and a pelvic expert radiologist.

Results: Most enrolled patients had squamous cell carcinoma and FIGO stage IIB disease, and initiated brachytherapy after the third week of pelvic external beam radiation. Gross tumor volume volume reduction from diagnostic MRI to $\mathrm{B} 1$ represented $61.9 \%$ and $75.2 \%$ of the initial volume, when measured by VE and VC, respectively. Only a modest volume reduction $(15-20 \%)$ was observed from B1 to B3.

Conclusions: The most expressive tumor shrinking occurred in the first three weeks of oncological treatment and was in accordance with gynecological examination. These findings may help in IGBT implementation.
\end{abstract}

Key words: brachytherapy, cervical cancer, cervix cancer, MRI.

\section{Purpose}

Uterine cervix cancer has a high incidence in developing countries and, despite the screening programs, diagnosis in an advanced stage is still common. In Latin America, incidence rates range from 20 to 80 per 100000 women per year [1], however, screening protocols are not available for all women, resulting in a small impact effect in mortality reduction. In Brazil, the National Cancer Institute estimates an incidence of 16,340 new cervix cancer cases in 2016 with an estimated risk of 15.85/100 000 women [2]. Fortunately, both prophylaxis and management of patients with uterine cervix cancer have improved in recent years. This evolution has been mostly triggered by the new development of targeted systemic therapies and by advances in tridimensional (3D) brachytherapy.

Compatible computed tomography (CT) and magnetic resonance (MRI) tandem, ring, and ovoids have enabled the acquisition of 3D pelvic images with the applicators placed in the treatment position, allowing a good perception of tumor volume and its relation to the applicators. To help clinicians move forward and standardize treatments in this new technique, guidelines in image-guided brachytherapy (IGBT) have been written [3]. It was demonstrated that tumor coverage with the conventional two-dimensional (2D) plan, based on semi-orthogonal radiographs, was suboptimal, especially for large lesions, and IGBT could achieve a better tumor coverage and reduce dose to normal tissue [4]. In fact, great outcomes were reported by the Vienna and Aarhus groups [5,6,7], suggesting the superiority of IGBT against conventional brachytherapy. Better toxicity profile, higher local control, and specific disease-free survival were also observed in these reports. Public health in developing countries has limited resources, and implementation of a new technology should be rationalized and confronted with its related 
costs. Despite the benefits associated with IGBT in cervix carcinoma treatment, MRI availability and costs in each fraction of brachytherapy plus staff time consumed to perform the treatment represent some of the limiting factors. Thus, there is a need for further progress with regards to patient stratification and procedure evaluation to leverage a more positive cost-effective ratio. In this scenario, the Vienna group $[5,6]$ has reported that patients who benefit the most from IGBT are those with locally advanced disease, particularly women with tumors larger than $5 \mathrm{~cm}$.

In 2010, our institution started a study for implementation of 3D IGBT. One of the goals was to optimize the resources for the procedure. In preparation to start an IGBT program, a tumor volume regression evaluation was done.

This study aimed to evaluate tumor regression kinetics pattern in patients with cervical cancer treated with chemoradiation.

\section{Material and methods}

This study was approved by the institutional ethical committee (Comissão de Ética para Análise de Projetos de Pesquisa - CAPPesq da Diretoria Clínica do HCFMUSP) and registered as: CAPPesq $\mathrm{n}^{\circ} 1100 / 08$. Before the beginning of the study, all patients had signed an informed consent.

From February 2010 to December 2011, fourteen patients with confirmed histological diagnosis of cervix squamous cell or adenocarcinoma were enrolled. Good performance status (ECOG $<3$ ), a pelvic MRI at diagnosis, and FIGO stages IB to IIIB were required for inclusion in this study.

Patients were recruited sequentially and treated in accordance to the institutional protocol, which comprises additional local staging with pelvic MRI, pelvic external beam radiation therapy (EBRT) with concomitant weekly cisplatin-based chemotherapy, and two-dimensional (2D) intracavitary high-dose-rate brachytherapy (HDRB). Pelvic EBRT was performed with 6 or 15 MV photons, in 25 fractions of 1.8 Gy each (45 Gy total dose) with a 4-field conformal box technique. Patients with involved parametria received a 14 Gy boost in 7 fractions with opposed anterior-posterior/posterior-anterior fields and central shielding. High-dose-rate brachytherapy was delivered during EBRT once a week in four fractions of 7 or $7.5 \mathrm{~Gy}$, prescribed at Manchester point A (7.5 Gy was prescribed for stage III patients). Dose constraints at ICRU 38 bladder and rectal points were limited to $65 \%$ of point A dose. In our practice, patients are routinely reassessed with gynecological exams after the second week of EBRT in order to evaluate local anatomy/geometry for brachytherapy. If brachytherapy is not performed, patients continue through weekly gynecological exams until proper geometry is achieved. Usually, these patients start brachytherapy after the end of the pelvic irradiation, and fractions are delivered twice a week in order to finish the whole treatment in less than 8 weeks. Chemotherapy consisted of weekly cisplatin $40 \mathrm{mg} / \mathrm{m}^{2}$ up to six cycles given concomitantly to radiotherapy. No chemotherapy or EBRT were allowed on the days of brachytherapy.
The brachytherapy protocol for implementation of 3D IGBT followed exactly the same schedule, except for the performance of MRI in the first and third HDRB fractions, and CT scans in the second and fourth HDRB fractions. Local anesthesia and conscious sedation were used for cervix dilation and placement of the CT-MR compatible tandem-ring applicator (Nucletron ${ }^{\circledR}$, an Elekta Company, Sweden), under ultrasound guidance. All patients were treated with standard 2D technique using semi-orthogonal pelvic radiographs. Plato ${ }^{\circledR}$ version 14.1 and subsequently Oncentra Masterplan ${ }^{\circledR}$ version 4.1 (Nucletron ${ }^{\circledR}$, an Elekta Company, Sweden) were the planning systems used for calculations. Magnetic resonance images, both at diagnosis and at brachytherapy, were performed with a 1.5 Tesla GE Signa HDxt scanner (GE Healthcare, Chalfont St. Giles, UK [a unit of General Electric Company] ${ }^{\circledR}$ ), with $3 \mathrm{~mm}$ slice thickness. The assessment of the target extension in all planes was performed in para-transverse, para-coronal, and sagittal planes oriented according to the uterine axis or the applicator, in T2-weighted FSE sequences, following the GEC-ESTRO recommendations [8]. In order to avoid bladder volume variations, all patients had their bladder emptied and subsequently filled with $50 \mathrm{ml}$ of saline solution before each image acquisition and treatment procedures.

Tumor (gross tumor volume [GTV], high-risk clinical target volume [HR-CTV], intermediate-risk clinical target volume [IR-CTV]) and organs at risk [OAR] - bladder, rectum and sigmoid) volumes delineation were performed on MRI studies using T2-weighted images, in accordance with the GEC-ESTRO recommendations [8]. Three independent senior radiation oncologists and one pelvic expert radiologist participated in volume delineation of each MRI image. Definitive volume was defined by agreement of at least two radiation oncologists and the radiologist.

Tumor volume was defined by slice-by-slice MRI contouring (VC) and also estimated by the ellipsoid formula (VE), which, is the product of the three largest diameters in three different plans (axial, sagittal, and coronal) of the MRI multiplied by $\pi / 6$, as already used by others [9].

VC and VE were calculated on MRI at diagnosis (D) and, in the first (B1) and third (B3) brachytherapy fractions. The kinetic of tumor reduction was then evaluated for each patient. Computed tomography images were not used for the matter of this report.

Descriptive analysis was performed to describe frequencies and interquartile intervals. Since data distribution was not normal, non-parametric tests were used for comparisons. Friedman test and Wilcoxon sign rank test evaluated tumor volumes regression. Spearman correlation test evaluated VC and VE. Statistical significance was set at $5 \%(p<0.05)$.

\section{Results}

\section{Patients characteristics}

One out of fourteen patients enrolled in this protocol was excluded due to psychiatric disorder, which disabled MRI to be performed during brachytherapy. 
Thus, thirteen women with 39 MRIs were the object of this study. Patients median age was 46 years (range 31 to 76 years) and all of them had ECOG performance status equal to zero. Nine patients had squamous cell histology and the others adenocarcinoma. Two patients presented FIGO stage IB, one stage IIA, nine were IIB, and one IIIB. Among IIB cases, two presented with enlarged pelvic lymph node considered to be positive.

\section{Treatment}

Radiotherapy started with a median delay of 49 days (range 10 to 102 days) from the date of diagnostic pelvic MRI, and the median start time between EBRT and the first brachytherapy fraction was 36 days (range 23 to 95 days). All patients received six cycles of cisplatin, except for one, who received five cycles. The entire oncological treatment was completed within a median time of 60 days (range: 47 to 83 days). Three patients presented more extended total treatment time mainly due to interruptions related to hematological toxicity of the chemoradiation schedule. Seven patients initiated HDRB between the third and fifth week of EBRT and six after 45 Gy EBRT to the pelvis.

All HDRB fractions were performed with tandem and ring applicator using the rectal retractor. One out of 13 patients had the ring diameter reduced from $34 \mathrm{~mm}$ to $30 \mathrm{~mm}$ along treatment; two patients had the tandem length shortened from $60 \mathrm{~mm}$ to $40 \mathrm{~mm}$, and in one patient, a different applicator angle was used along brachytherapy fractions.

\section{Volumes analysis}

Figure 1 shows tumor volume regression throughout oncological treatment. Most of the gross tumor volume reduction occurred from the diagnostic MRI to the first brachytherapy fraction (Figure 2).

Tables 1 and 2 compare tumor volumes estimated by the ellipsoid formula (VE) and calculated by MRI contouring (VC) in three different moments: D, B1, and B3. A mean absolute volume reduction of approximately $16 \mathrm{cc}$ was observed between diagnosis and the first brachytherapy fraction by both calculation methods. This volume reduction represented $61.9 \%$ and $75.2 \%$ of the diagnostic GTV, when measured by VE and VC, respectively. We compared tumor kinetics' of the seven patients in whom brachytherapy was started before the end of EBRT with the other six where HDRB started after completion of EBRT (45 Gy). VE and VC at diagnosis were similar in both groups ( $p=0.173$ and 0.686 , respectively), as the

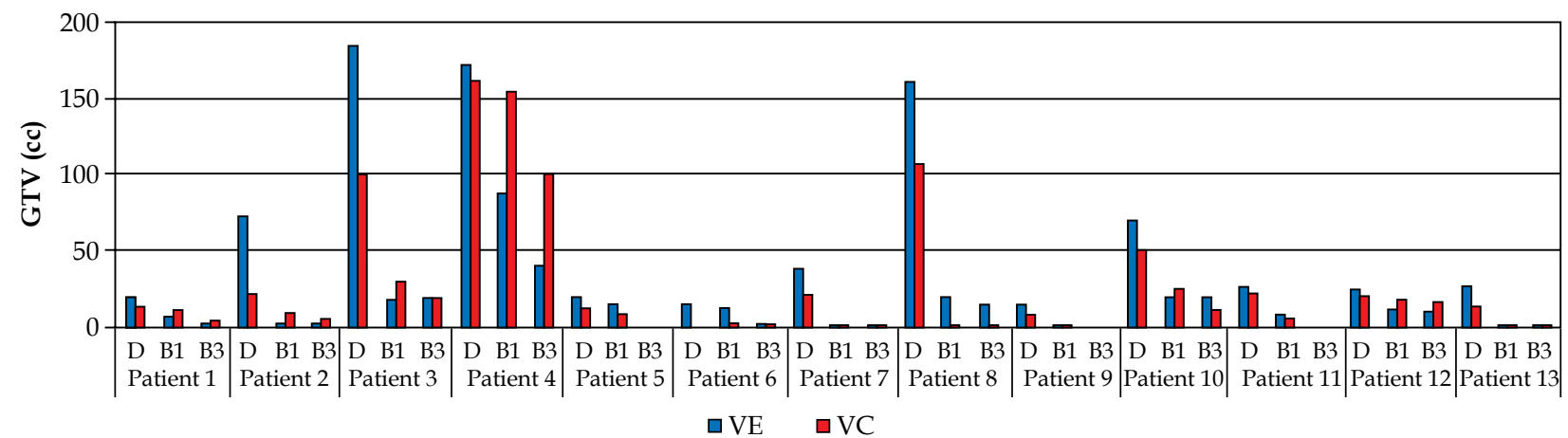

Fig. 1. Gross tumor volumes (GTV) in each patient calculated by the ellipsoid formula (VE) and slice-by-slice contouring (VC) at magnetic resonance imaging at diagnosis (D), first (B1), and third (B3) brachytherapy fractions

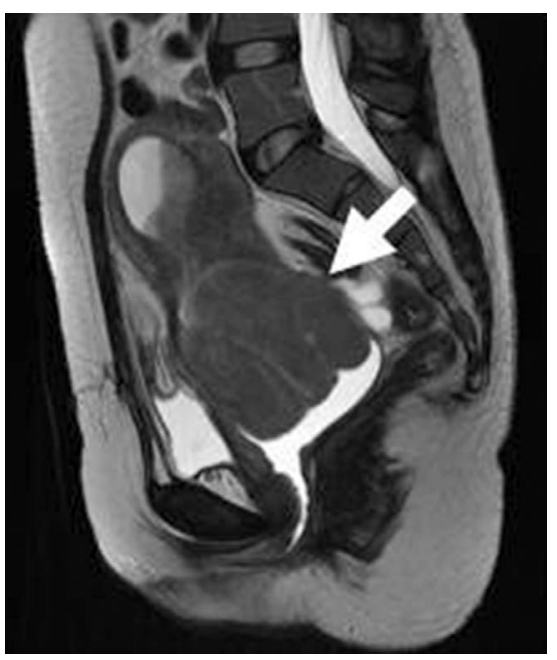

B1

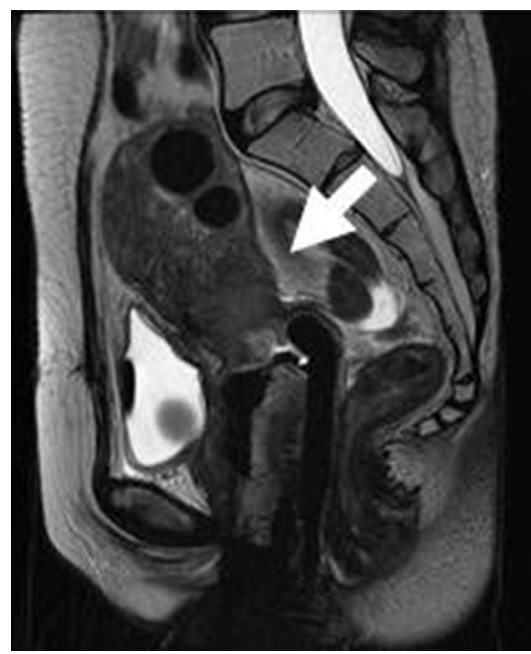

B3

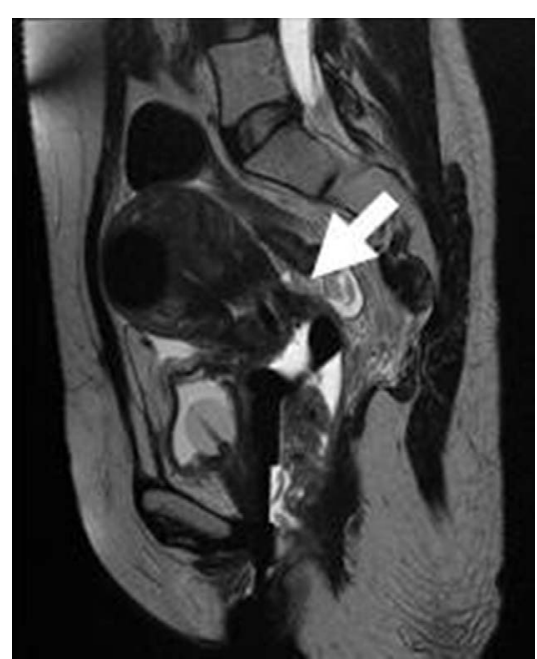

Fig. 2. Magnetic resonance (T2-weighted images, sagittal view) of patient \#3 that presented good response (white arrow). $\mathrm{D}$ - at diagnosis, B1 - at first brachytherapy fraction, and B3 - at third brachytherapy fraction, respectively 
Table 1. Ellipsoid formula (VE) estimated volume along treatment: at diagnosis $\left(\mathrm{GTV}_{\mathrm{D}}\right)$, first $\left(\mathrm{GTV}_{\mathrm{B} 1}\right)$, and third $\left(\mathrm{GTV}_{\mathrm{B} 3}\right)$ brachytherapy fractions

\begin{tabular}{|c|c|c|c|c|}
\hline VE & Median (IQ) (cc) & Shrinkage (\%) & $p^{*}$ & $p^{\#}$ \\
\hline$G_{T} V_{D}$ & $25.5(17.4-116.8)$ & 61.9 & 0.001 & 0.0001 \\
\hline $\mathrm{GTV}_{\mathrm{B} 1}$ & $9.7(1.3-18.0)$ & & & \\
\hline $\mathrm{GTV}_{\mathrm{B} 1}$ & $9.7(1.3-18.0)$ & 54.7 & 0.008 & \\
\hline $\mathrm{GTV}_{\mathrm{B} 3}$ & $4.4(0.05-16.6)$ & & & \\
\hline $\mathrm{GTV}_{\mathrm{D}}$ & $25.5(17.4-116.8)$ & 82.7 & 0.005 & \\
\hline $\mathrm{GTV}_{\mathrm{B} 3}$ & $4.4(0.05-16.6)$ & & & \\
\hline
\end{tabular}

*Wilcoxon signed rank test, ${ }^{\text {FFriedman test }}$ IIQ - interquartile range

tumor regression pattern evaluated by VE and VC at B1 ( $p=0.116$ and 0.753 , respectively).

Mean overall tumor shrinkage, evaluated by VE and VC, from the diagnostic MRI to third brachytherapy fraction was $82.7 \%$ and $89.0 \%$, respectively. Thus, only

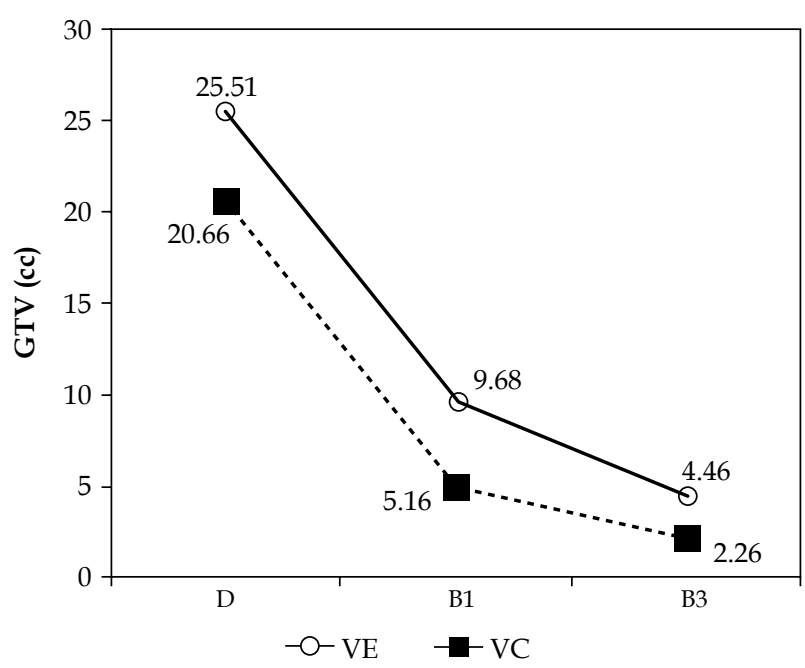

Fig. 3. Tumor median volumes calculated by ellipsoid formula (VE) and slice-by-slice delineation (VC) at magnetic resonance imaging diagnosis (D), first (B1), and third (B3) brachytherapy fractions
Table 2. Slice-by-slice delineation volume (VC) along treatment: at diagnosis $\left(G V_{D}\right)$, first $\left(G T V_{B 1}\right)$, and third $\left(\mathrm{GTV}_{\mathrm{B} 3}\right)$ brachytherapy fractions

\begin{tabular}{|c|c|c|c|c|}
\hline VC & Median (IQ) (cc) & Shrinkage (\%) & $p^{*}$ & $p^{\#}$ \\
\hline GTV $_{D}$ & $21.0(11.3-103.9)$ & 75.2 & 0.001 & 0.0001 \\
\hline GTV $_{B 1}$ & $5.2(0.5-18.9)$ & & & \\
\hline $\mathrm{GTV}_{\mathrm{B} 1}$ & $5.2(0.5-18.9)$ & 55.8 & 0.008 & \\
\hline GTV $_{\text {B3 }}$ & $2.3(0-14.5)$ & & & \\
\hline $\mathrm{GTV}_{\mathrm{D}}$ & $21.0(11.3-103.9)$ & 89.0 & 0.005 & \\
\hline GTV $_{\text {B3 }}$ & $2.3(0-14.5)$ & & & \\
\hline
\end{tabular}

*Wilcoxon signed rank test, " Spearman's correlation test $1 Q$ - interquartile range

a modest reduction of $15-20 \%$ of the GTV volume was seen between the first and third HDRB fractions. Again, no statistically significant differences were observed in tumor kinetics' when patients were evaluated according to the dose of EBRT received at B3.

No significant correlation was found between VE and $\mathrm{VC}$ at the diagnostic MRI. VE calculation overestimated the contoured volume. However, VE and VC were statistically significant correlated in both B1 and B3, with a higher correlation index observed in smaller tumor volumes (Figure 3, Table 3).

High-risk clinical target volume in B1 and B3 were also evaluated. VC presented similar volumes in B1 and B3, with a median volume estimated as $21.4 \mathrm{cc}$ (7-151 cc) and $21.6 \mathrm{cc}$ (11-97 cc), respectively. Both volumes were highly correlated $($ rho $=0.891 / p=0.001$; Spearman correlation test).

\section{Discussion}

Brachytherapy is fundamental in the treatment of advanced uterine cervix cancer because improves overall survival [10]. Image-guided brachytherapy is a recent evolution from 2D brachytherapy and allows clinicians to optimize treatment to a visible and measurable gross tumor volume. This is particularly useful when we are faced with large tumors, as often diagnosed in developing countries [11]. Thus, dose prescription and isodose

Table 3. Tumor volumes comparisons calculated by VE and VC at diagnosis (GTV $)$, first $\left(G_{T} V_{B 1}\right)$, and third $\left(\mathrm{GTV}_{\mathrm{B} 3}\right)$ brachytherapy fractions

\begin{tabular}{|c|c|c|c|c|c|c|}
\hline \multicolumn{2}{|c|}{ Volume } & \multirow{2}{*}{$\frac{\text { Median (IQ) (cc) }}{25.5(17.4-116.8)}$} & \multirow{2}{*}{$\frac{\text { Range (cc) }}{12.6-184.2}$} & \multirow{2}{*}{$\frac{p^{*}}{0.023}$} & \multirow{2}{*}{$\frac{\text { rho }^{\#}}{0.505}$} & \multirow{2}{*}{$\frac{p^{\#}}{0.078}$} \\
\hline $\mathrm{GTV}_{\mathrm{D}}$ & VE & & & & & \\
\hline & VC & $21.0(11.3-103.9)$ & $7.6-164.8$ & & & \\
\hline \multirow[t]{2}{*}{$\mathrm{GTV}_{\mathrm{B} 1}$} & VE & 9.7 (1.3-18.0) & $0.5-86.3$ & 0.650 & 0.604 & 0.029 \\
\hline & VC & $5.2(0.5-18.9)$ & $0.0-151.5$ & & & \\
\hline \multirow[t]{2}{*}{$\mathrm{GTV}_{\mathrm{B} 3}$} & VE & $4.4(0.05-16.6)$ & $0.0-38.2$ & 0.678 & 0.726 & 0.017 \\
\hline & VC & $2.3(0-14.5)$ & $0-97.7$ & & & \\
\hline
\end{tabular}

*Wilcoxon signed rank test, "Spearman's correlation test

$1 Q$ - interquartile range, VE - ellipsoid formula, VC - slice-by-slice delineation volume 
shaping can be individualized in accordance to specific tumor, the cervix and suspicious extra-cervical residual tissues while taking the initial tumor shape and size into account, and OAR anatomy. As expected, toxicity profile related to this technique is milder in comparison to that achieved with 2D brachytherapy $[12,13,14]$. Moreover, absolute local control can be approximately $20 \%$ higher than published results using 2D technique in the literature resulting in an absolute 3 -year cancer specific survival benefit of 13 to $30 \%$ in patients with tumors larger than $5 \mathrm{~cm}[6,12,13]$.

In order to perform IGBT, a 3D pelvic image acquisition with the applicators in place is necessary. Both CT and MRI are useful in this setting but MRI provides a better soft tissue contrast and is superior in gross tumor volume definition $[15,16]$. However, MRI is more expensive than CT and its image acquisition is longer, thus limiting its use in developing countries where cervix cancer has a high incidence.

In the present study, tumor kinetics' along cancer treatment was evaluated by serial MRIs. Around 70\% tumor volume reduction was achieved from diagnosis to the first HDRB fraction, which is in accordance to others findings [17]. Considering that in the present patient cohort there was a median delay of 49 days from the diagnostic MRI to the initiation of brachytherapy, it is possible that the relative volume reduction between tumor volume prior to EBRT and the first fraction of brachytherapy would be even larger than $70 \%$. This delay may represent a limitation of our study. Nevertheless, this important volume reduction in the first weeks of EBRT suggests that IGBT could start, at least after the third week of EBRT, since after this period, a substantial regression of tumor's volume is not expected. One may argue that a maximum tumor regression is observed at the end of EBRT, and thus, IGBT should be performed at this point, making our analysis irrelevant. However, in this scenario, brachytherapy would preferably be delivered in two fractions per week in order to avoid an extended total treatment time over 56 days. This may represent a burden in very busy departments like ours, justifying why efforts are done to start brachytherapy during EBRT. Nevertheless, even in this small cohort of patients, median overall treatment time was 60 days, slightly higher than the desirable.

Most patients were submitted to brachytherapy after the third week of EBRT according to clinical evaluation by weekly gynecological exams. This was compatible with the volume regression observed by MRI. For smaller tumors, volume variation was obviously smaller, and brachytherapy was performed earlier with no major impact in HR-CTV delineation, since this CTV comprehends the entire cervix, harboring or not the disease, and treatment volumes may not change significantly between HDR fractions [3]. This may be the case of most patients in this study, in which a relatively small disease burden was found at diagnosis (median GTVD by VC = $21 \mathrm{cc}$, Table 2). For larger tumors where tumor regression has a major impact in volume delineation, postponing the procedure would be more useful. Thus, weekly gynecological evaluation in our experience was helpful to manage the timing for the first brachytherapy fraction in this setting. This represents one of the highlights of our study.

On the other hand, in this group of patients, while performing images enabling 3D procedures, HDRB was delivered with 2D based calculation. This is another limitation of our study, since no dose-volume parameters were considered in this analysis. Nevertheless, during this period, it was possible to establish a new routine for applicator placement and fixation (with only local anesthesia and sedation), to establish a method for transportation of the patient to the imaging rooms, and to training of tumor volumes contouring in MRI, as well as develop all the process for implementation of an IGBT programme [18]. The tumor shrinking kinetics approach was used to establish better timing for the procedure and to possibly save some resources. Performing MRI in a timely manner may allow its use only in the first IGBT fraction, followed by $\mathrm{CT}$ based insertions in the subsequent fractions, as already proposed by others $[15,19]$. Since CT contoured volumes are known to overestimate the target [15], tumor regression evaluation may be helpful to achieve more accurate delivery of CT-based IGBT fractions. Possibly, following this strategy, a good ratio between MRI benefits and costs could be found and this resource be more often allocated for treatment of cervical cancer patients in developing countries.

The main objective of this study was the evaluation of the tumor shrinkage during treatment. In addition, the feasibility of the procedure was evaluated for further implementation of the method in the institution. No sample size was calculated upfront, neither a maximum time between the diagnosis MRI and the initiation of EBRT or HDRB was required. We are aware that the small number of patients and the variation of time elapsed since diagnosis MRI to treatment also represent limitations of this study. On the other hand, our results may better reflect obstacles and difficulties to implement the technology at our institution, in a group of patients treated according to our daily routine. But, even with these limitations, our results were in agreement with others that looked at tumor shrinkage during irradiation $[17,20,21]$. In these studies, the effects of EBRT alone or brachytherapy on tumor shrinkage were evaluated. In our study, tumor regression was evaluated in a different treatment schedule with HDRB performed during chemoradiation. Brachytherapy when performed during chemoradiation may present an even higher tumor regression and the influence of this combination may be explored in further studies.

A parallel result of this study was the comparison between the different methods of volume evaluation throughout treatment. Not surprisingly, the ellipsoid formula overestimated the gross tumor volume on the staging MRI. As expected, large tumor volumes with irregular shapes are not well estimated by VE that takes into consideration spheroid shapes using only three planes coordinates. As tumor shrinks, the estimated VE better correlates with VC. These findings are in accordance with Mayr et al. [9], and may indicate that the results of Dimopoulos et al. [17], based on the ellipsoid formula, may either under or overestimate tumor regression. Despite these differences between tumor volumes measured by 
VE and VC due to limitations of the ellipsoid formula, both methods were consistent and presented a similar pattern of tumor volume reduction along treatment as shown in Figure 2. In addition, the volumes were defined by a consensus of at least three experienced professionals among radiologists and radiation oncologists, which gives reliability to the measures performed, and prevents delineation uncertainties [22]. These findings should be validated with a larger number of patients.

Not in the scope of this study but worth to comment, tumor regression during treatment may also be used as a prognostic factor $[9,23,24,25]$, and as a very useful tool in order to pre-plan the brachytherapy procedure - intracavitary only or associated with interstitial implant [26].

The level of MRI utilization in IGBT depends on the infrastructural capabilities of individual centers, ranging from no use at all to repetitive imaging during EBRT and each IGBT fraction [27]. Despite the large number of uterine cervix cancer cases at our institution, only two patients per month could be recruited due to the overloading of the MRI agenda, which reflects the difficulties faced by our country, even in a reference center. For this same reason, studies looking at resource's optimization are warranted and welcome in this context.

The benefits of the better use of technical resources come with the charge of personnel training and the more time-consuming of the whole treatment process. The later represents a limitation for the routine use of 3D IGBT in developing countries where radiation oncology departments are busy, usually with waiting lists, and with limited human and technical resources [28]. Currently, our department is able to perform only one case per month (one fraction/week) of intracavitary 3D IGBT due to logistical issues. No interstitial implants are being performed in this context yet. Thus, understanding which patients would benefit the most from these technique is desirable, until it becomes possible to offer this technology for all patients. The results of this study helped us to establish a routine in the department, even for $2 \mathrm{D}$ brachytherapy treatments, in which patients with indication of HDRB for cervix cancer should start the procedure after the third week of treatment. This understanding is important to build a resource-saving strategy and successfully allocate limited available funds in undeveloped countries. MRI-guided brachytherapy in the first fraction followed by CT-based brachytherapy for the subsequent fractions may lead to more accurate treatment, if steep tumor volume reduction is waited to occur.

\section{Conclusions}

Most expressive tumor shrinkage occurs during the first three weeks of chemoradiation. Clinical evaluation by gynecological exams helped to determine the optimal time for brachytherapy and were in accordance with imaging exams. This finding may help in the decision making process of 3D-IGBT implementation.

\section{Disclosure}

Authors report no conflict of interest.

\section{References}

1. Villa L. Cervix cancer in Latin America and the Caribbean: the problem and the way to solutions. Cancer Epidemiol Biomarkers Prev 2012; 21: 1409-1413.

2. Brasil, Ministério da Saúde, INCA. Estimativa 2016: incidência de cânc er no Brasil/Instituto Nacional de Câncer José Alencar Gomes da Silva - Rio de Janeiro: INCA, 2015. Available at: www.inca.gov.br

3. Haie-Meder C, Pötter R, Van Limbergen E et al. Recommendations from Gynaecological (GYN) GEC-ESTRO Working Group (I): concepts and terms in 3D image based 3D treatment planning in cervix cancer brachytherapy with emphasis on MRI assessment of GTV and CTV. Radiother Oncol 2005; 74: 235-245.

4. Zwahlen D, Jezioranski K, Chan P et al. Magnetic resonance imaging-guided intracavitary brachytherapy for cancer of the cervix. Int J Radiat Oncol Biol Phys 2009; 74: 1157-1164.

5. Pötter R, Dimopoulos J, Georg P et al. Clinical impact of MRI assisted dose volume adaptation and dose escalation in brachytherapy of locally advanced cervix cancer. Radiother Oncol 2007; 83: 148-155.

6. Pötter R, Georg P, Dimopoulos JC et al. Clinical outcome of protocol based image (MRI) guided adaptive brachytherapy combined with 3D conformal radiotherapy with or without chemotherapy in patients with locally advanced cervix cancer. Radiother Oncol 2011; 100: 116-123.

7. Lindegaard JC, Fokdal LU, Nielsen SK et al. MRI-guided adaptive radiotherapy in locally advanced cervical cancer from a Nordic perspective. Acta Oncol 2013; 52: 1510-1519.

8. Dimopoulos JC, Petrow P, Tanderup K et al. Recommendations from Gynaecological (GYN) GEC-ESTRO Working Group (IV): Basic principles and parameters for MR imaging within the frame of image based adaptive cervix cancer brachytherapy. Radiother Oncol 2012; 103: 113-122.

9. Mayr NA, Taoka T, Yuh WT et al. Method and timing of tumor volume measurement for outcome prediction in cervical cancer using magnetic resonance imaging. Int J Radiat Oncol Biol Phys 2002; 52: 14-22.

10. Han K, Milosevic M, Fyles A et al. Trends in the utilization of brachytherapy in cervical cancer in the United States. Int J Radiat Oncol Biol Phys 2013; 87: 111-119.

11. Thuler LCS, de Aguiar SS, Bergmann A. Determinantes do diagnóstico em estádio avançado do câncer do colo do útero no Brasil. Rev Bras Ginecol Obstet 2014; 36: 237-243 [Article in Portuguese].

12. Pötter R, Knocke TH, Fellner $C$ et al. Definitive radiotherapy based on HDR brachytherapy with iridium 192 in uterine cervix carcinoma: report on the Vienna University Hospital findings (1993-1997) compared to the preceding period in the context of ICRU 38 recommendations. Cancer Radiother 2000; 4: 159-172.

13. Tan LT, Coles CE, Hart C et al. Clinical impact of computed tomography-based image-guided brachytherapy for cervix cancer using the tandem-ring applicator - the Addenbrooke's experience. Clin Oncol (R Coll Radiol) 2009; 21: 175-182.

14. Kang HC, Shin KH, Park SY et al. 3D CT-based high-doserate brachytherapy for cervical cancer: clinical impact on late rectal bleeding and local control. Radiother Oncol 2010; 97: 507-513.

15. Beriwal S, Kannan N, Kim H et al. Three-dimensional high dose rate intracavitary image-guided brachytherapy for the treatment of cervix cancer using a hybrid magnetic resonance imaging/computed tomography approach: feasibility and early results. Clin Oncol (R Coll Radiol) 2011; 23: 685-690.

16. Dimopoulos JC, Schard G, Berger D et al. Systematic evaluation of MRI findings in different stages of treatment of cervix 
cancer: potential of MRI on delineation of target, pathoanatomic structures, and organs at risk. Int J Radiat Oncol Biol Phys 2006; 64: 1380-1388.

17. Dimopoulos JC, Schirl G, Baldinger A et al. MRI assessment of cervical cancer for adaptive radiotherapy. Strahlenther Onkol 2009; 185: 282-287.

18. Owrangi AM, Prisciandaro JI, Soliman A et al. Magnetic resonance imaging-guided brachytherapy for cervical cancer: initiating a program. J Contemp Brachytherapy 2015; 7: 417-422.

19. Nesvacil N, Pötter R, Sturdza A et al. Adaptive image guided brachytherapy for cervical cancer: a combined MR/CT-planning technique with MRI only at first fraction. Radiother Oncol 2013; 107: 75-81.

20. Mayr NA, Magnotta VA, Ehrhardt JC et al. Usefulness of tumor volumetry by magnetic resonance imaging in assessing response to radiation therapy in carcinoma of the uterine cervix. Int J Radiat Oncol Biol Phys 1996; 35: 915-924.

21. Hatano K, Sekiya Y, Araki H et al. Evaluation of the therapeutic effect of radiotherapy on cervical cancer using magnetic resonance imaging. Int J Radiat Oncol Biol Phys 1999; 45: 639-644.

22. Petrič P, Hudej R, Rogelj $P$ et al. Uncertainties of target volume delineation in MRI guided adaptive brachytherapy of cervix cancer: a multi-institutional study. Radiother Oncol 2013; 107: 6-12.

23. Mayr NA, Yuh WT, Taoka T et al. Serial therapy-induced changes in tumor shape in cervical cancer and their impact on assessing tumor volume and treatment response. AJR Am J Roentgenol 2006; 187: 65-72.

24. Mayr NA, Wang JZ, Lo SS et al. Translating response during therapy into ultimate treatment outcome: a personalized 4-dimensional MRI tumor volumetric regression approach in cervical cancer. Int J Radiat Oncol Biol Phys 2010; 76: 719-727.

25. Wang JZ, Mayr NA, Zhang D et al. Sequential magnetic resonance imaging of cervical cancer: the predictive value of absolute tumor volume and regression ratio measured before, during, and after radiation therapy. Cancer 2010; 116: 5093-5101.

26. Fokdal L, Tanderup K, Hokland SB et al. Clinical feasibility of combined intracavitary/interstitial brachytherapy in locally advanced cervical cancer employing MRI with a tan$\mathrm{dem} /$ ring applicator in situ and virtual preplanning of the interstitial component. Radiother Oncol 2013; 107: 63-68.

27. Petric $\mathrm{P}$, Mohammed-Al-Hammadi N. MRI findings at image guided adaptive cervix cancer brachytherapy: radiation oncologist's perspective. J Contemp Brachytherapy 2014; 6: 215-222.

28. Moraes FY, Marta GN, Hanna SA et al. Around the globe: Brazil's challenges and opportunities. Int J Radiat Oncol Biol Phys 2015; 92: 707-712. 